\title{
Feasibility and Efficacy of Inspiratory Muscle Training in Patients with Head and Neck Cancer Receiving Concurrent Chemoradiotherapy
}

\author{
Prina Vira ${ }^{1}$, Stephen R Samuel ${ }^{1 *}$, Santosh Rai PV ${ }^{2}$, PU Prakash Saxena ${ }^{3}$, Sampath \\ Kumar Amaravadi ${ }^{1,4}$, Nagaraja Ravishankar ${ }^{5}$, Diwakar D Balachandran ${ }^{6}$
}

\begin{abstract}
Objectives: Patients with head and neck cancer (HNC) undergoing concurrent chemoradiotherapy (CCRT) often experience pulmonary symptoms. This study evaluated if a 7-week inspiratory muscle training (IMT) program during CCRT is feasible, adherent, and safe in patients with HNC. This study also evaluated the effect of IMT on diaphragm thickness, mobility, and cardiorespiratory parameters in patients with HNC receiving CCRT. Methods: Ten participants with advanced stage HNC receiving CCRT were recruited for the study. Feasibility, adherence, and safety of the intervention were the primary outcomes. Changes in diaphragm thickness and mobility, maximal inspiratory pressure, maximal expiratory pressure, forced vital capacity, forced expiratory volume in first second and functional capacity using 6-MWT were measured at baseline and post 7 weeks of CCRT. IMT was performed at one session per day for 5 days a week for 7 weeks. Eight sets of two minutes of inspiratory manoeuvres with one minute rest period between them with intensity of 40\% MIP were given. Results: Ten participants were included in this study out of the 13 patients screened, indicating the feasibility to be $76.9 \%$. Participants completed a total of 260 training sessions out of the 350 planned sessions denoting the adherence level as $74 \%$. Diaphragm thickness and MEP remained significantly unchanged while significant decline was seen in diaphragm mobility, MIP,FVC, FEV1 and 6-MWD at the end of 7 weeks. No adverse events were reported following the intervention. Conclusion: Inspiratory muscle training did not show significant effect on the diaphragm thickness, mobility, and cardiorespiratory parameters; however, it was feasible, adherent, and safe in patients with HNC receiving CCRT.
\end{abstract}

Keywords: Head and neck cancer- inspiratory muscle training- diaphragm- respiratory muscle strength

Asian Pac J Cancer Prev, 22 (12), 3817-3822

\section{Introduction}

Patients with head and neck cancer (HNC) often experience smoking related respiratory symptoms (Petrar et al., 2012; Silverman et al., 2020). Due to common risk factor of tobacco smoking, patients with HNC demonstrate obstructive lung defects on pulmonary function testing and may have undiagnosed underlying pulmonary conditions (Volpe et al., 2011; Gottlieb et al., 2015). Furthermore, smoking increases the oxidative stress on the respiratory epithelium, disrupts the mucociliary clearance and impairs the immunity, predisposing them to lung infections (Silverman et al., 2020).

Decreased inspiratory muscle strength, diaphragmatic immobility and atrophy have been shown to occur in patients with head and neck cancer following neck dissection, leading to impaired gas exchange and poor cough effort. Temporary inhibition of phrenic nerve, physical inactivity and reduced protein calorie intake are potential mechanisms for these abnormalities (Santana et al., 2018). However, these findings were shown to occur in patients following surgical interventions and therefore cannot necessarily be extended to patients receiving concurrent chemoradiotherapy (CCRT).

Chemoradiation causes various treatment-related side effects such as muscle wasting, sarcopenia, cachexia, and disuse muscle atrophy affecting both peripheral and inspiratory muscles (Nakano et al., 2017; Davis and Panikkar, 2019). Inspiratory muscles are known as 'skeletal muscles' but they vary in their structural and

${ }^{1}$ Department of Physiotherapy, Kasturba Medical College, Mangalore, Manipal Academy of Higher Education, Manipal, India. ${ }^{2}$ Department of Radiology, Kasturba Medical College, Mangalore, Manipal Academy of Higher Education, Manipal, India. ${ }^{3}$ Department of Radiation Oncology, Kasturba Medical College, Mangalore, Manipal Academy of Higher Education, Manipal, India. ${ }^{4}$ Department of Physiotherapy, College of Health Sciences, Gulf Medical University, Ajman, United Arab Emirates. ${ }^{5}$ Department of Biostatistics, Vallabhbhai Patel Chest Institute, University of Delhi, Delhi - 110007, India. ${ }^{6}$ Department of Pulmonary Medicine, The University of Texas MD Anderson Cancer Center, Houston, Texas, USA. *For Correspondence: stephen.samuel@manipal.edu 
functional properties (Merrell and Kardon, 2013). The, diaphragm, being the primary inspiratory muscle, has shown to undergo myocytic changes in elderly population causing dysfunction due to age-related sarcopenia. Also, patients with heart failure (HF) showed diaphragmatic abnormalities occurring earlier and to a larger extent than the peripheral muscles (Kelley and Ferreira, 2017). A recent study demonstrated sarcopenia in Indian patients with HNC undergoing CCRT that suggested this as a possible mechanism for decreased thickness and excursion of diaphragm in this population (Chauhan et al., 2020). All these factors lead to pulmonary dysfunction impacting their physical activity and cardiorespiratory fitness. (Roberts et al., 2013; Fields et al., 2019). A recent case report showed significant decrease in diaphragm mobility, thickness, respiratory pressures, and exercise capacity in a patient with HNC receiving CCRT (D’Souza et al., 2020).

Inspiratory Muscle Training (IMT) increases the inspiratory muscle strength by increasing the proportion of fatigue-resistant diaphragmatic fibres thereby, decreasing the work of breathing and improving respiratory reserves. (Frownfelter, 1987). It has shown to be effective in elderly population (Souza et al., 2014), in patients with Chronic Obstructive Pulmonary Disease (Beaumont et al., 2018), HF (Wenzel et al., 2013), oesophageal (van Adrichem et al., 2014) and lung cancers (Lai et al., 2017). IMT as an intervention has not been studied in patients with HNC receiving CCRT, making it imperative to investigate the feasibility, adherence, and safety and efficacy of this program on diaphragm thickness, diaphragm mobility and cardiorespiratory parameters was the secondary aim of the study.

\section{Materials and Methods}

\section{Study design and Participants}

This was a prospective pilot study approved by the Institutional Ethics Committee (IEC KMC MLR 11-19/572). The study was conducted at a tertiary care university hospital. Patients above 18 years of age with locally advanced primary tumour residing in head and neck region of stage III and IV, receiving CCRT were included in the study. The patients excluded were those with HNC previously treated with surgical interventions, severe trismus, artificial airways and any previous diaphragmatic dysfunction, or neuromuscular disease which could cause an impact on the evaluation. The samples were recruited from February 2020 to March 2021. The primary outcome was to evaluate feasibility, adherence, and safety of the intervention. The secondary outcome was to evaluate changes in diaphragm thickness, diaphragm mobility at quiet and deep breathing, respiratory muscle strength i.e., Maximal Inspiratory Pressure (MIP) and Maximal Expiratory Pressure (MEP), pulmonary function i.e., Forced Vital Capacity (FVC) and Forced Expiratory Volume in first second (FEV1) and functional capacity.

\section{Procedure}

Once the purpose of the study was explained to the participant, a written informed consent was obtained. The study participants were admitted in the hospital for
7 weeks while they received CCRT, 70 Gy at a dose of 2 Gy per day in 35 fractions with concurrent cisplatin (40 $\mathrm{mg} / \mathrm{m}^{2} / \mathrm{BSA}$ ) as prescribed by the Oncologist. All the outcome measures were evaluated before the start of CCRT (at baseline) and after 7 weeks of CCRT.

\section{Intervention}

The participants performed the IMT intervention over 7 weeks of their hospital stay using the Threshold IMT device (Philips MAS Respironics ${ }^{\circledR}$ ) with a frequency of one session per day for 5 days a week. The participants performed eight sets of two minutes of inspiratory manoeuvres with one minute rest period between them. The initial intensity was adjusted to $40 \%$ of the MIP measured during the baseline evaluation. The load intensity increment was performed weekly depending on their rate of perceived exertion (RPE) i.e., if RPE was $<5$ on modified Borg's scale, the load was increased by $5 \%$ every week.(Souza et al., 2014) The intervention was supervised by a physiotherapist and the sessions were conveniently scheduled according to timings of the patient's other tests, chemo/radiotherapy sessions, and meals.

\section{Outcome measures \\ Feasibility, adherence, and safety}

Feasibility of the study was calculated by dividing the participants to the total number of eligible patients, and considered adequate if at least $60 \%$ of eligible patients participated. Adherence to the intervention was calculated by dividing the number of sessions performed to the total number of programmed sessions. At least $70 \%$ of the potential sessions performed was considered as a good adherence level. Adverse events were monitored according to the Common Terminology Criteria for Adverse Events (CTCAE) v4.0. If less than 5\% of adverse events were observed during or after the intervention, the IMT protocol was considered as safe (de Almeida et al., 2020).

\section{Diaphragm Thickness and Mobility}

Diaphragm thickness was evaluated with the ultrasound B-mode by placing the linear transducer from the middle of the pleural line to the middle of the peritoneal line over the diaphragm zone of apposition, close to the costophrenic angle between the right anterior and medial axillary lines. The thickness was measured at Functional Residual Capacity (FRC) and then at Total Lung Capacity (TLC) and the average value of three consecutive measurements was recorded. Diaphragm mobility was evaluated by placing a convex transducer pointing medially in the anterior subcostal region between the midclavicular and anterior axillary lines to visualize the posterior third of the hemi diaphragm with B-mode of the ultrasound. Then using the ultrasound M-mode the amplitude of diaphragmatic excursion was measured during quiet breathing and deep breathing (Ueki et al., 1995; Testa et al., 2011). The average value of three consecutive measurements were recorded. The procedure was carried out by an experienced radiologist in the hospital. 


\section{Respiratory Muscle Strength}

MIP and MEP were measured using a portable hand-held mouth respiratory pressure meter (MicroRPM, CareFusion Micro Medical, Kent, UK). Nasal clip was used by the participants during all the manoeuvres. MIP was measured by instructing the participant to perform maximal inspiration up to TLC, starting from residual volume (RV). MEP was measured by instructing the participant to perform maximal expiration starting from TLC. Three measurements were taken over 1 minute interval. Highest value among the three were recorded (Steier et al., 2007).

\section{Pulmonary Function Test}

The pulmonary function tests were performed using a portable spirometer (COSMED Portable Diagnostic Spirometer Machine ${ }^{\circledR}$, Medical Technologies, Inc. Massachusetts, USA) in accordance with the standard guidelines of American Thoracic Society/European Respiratory Society. FVC and FEV1 measures were taken and best of the three manoeuvres were recorded (Graham et al., 2019).

\section{Functional Capacity}

It was measured by 6-Minute Walk Test following the standard guidelines of American Thoracic Society (Brooks et al., 2003).

\section{Statistical Analysis}

The collected data was entered into Statistical Package for Social Science (SPSS) for Windows, version 26. Data pertaining to all the outcome variables was found to be normally distributed. Therefore, mean and standard deviation were used as descriptive statistics. Paired $t$ test was performed to determine whether the changes in the outcome variables over the two end points (i.e., at the baseline and at the seventh week) were statistically significant. A "p" value of $<0.05$ was considered as statistically significant in all the analyses.

\section{Results}

Patients were screened for eligibility from February 2020 to March 2021. It must be noted that the study started during the COVID-19 pandemic outbreak and hence we faced challenges such as national lockdown, low influx of patients and temporary suspension of the ongoing research to prevent the spread of COVID-19. All these factors resulted in a lower sample size of the study. After screening, 13 patients met the inclusion criteria out of which, three declined to participate. Accordingly, 10 participants were included in this study, indicating the feasibility to be $76.9 \%$. The demographic characteristics of the participants are listed in the Table 1. No participants withdrew from the ongoing study, but one participant was unable to complete the study due to the sudden

Table 1. Demographic Characteristics

\begin{tabular}{lc}
\hline Patients included & 10 \\
\hline Age & $52.70 \pm 11.19$ \\
Males/Females & $8(80 \%) / 2(20 \%)$ \\
BMI & $20.78 \pm 2.9$ \\
Cancer site & Buccal Mucosa (4\%) \\
& Piriformis fossa (10\%) \\
& Tongue (30\%) \\
& Floor of mouth (10\%) \\
& Oropharynx $(10 \%)$ \\
Clinical Stage & III $(10 \%)$ \\
& IV A (50\%) \\
Risk factors & IV B (40\%) \\
& Smoking $(50 \%)$ \\
& Tobacco chewing $(30 \%)$ \\
\hline
\end{tabular}

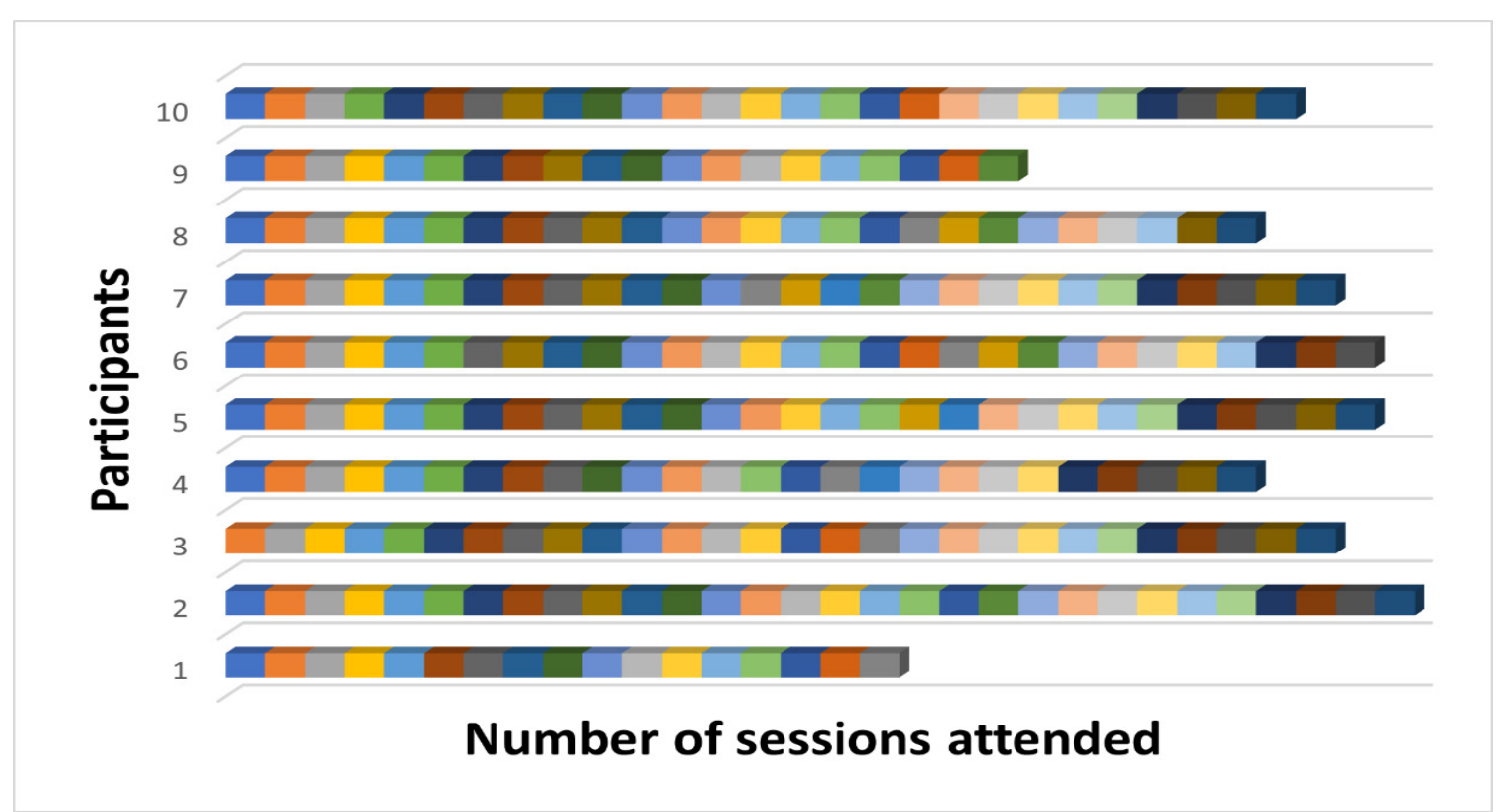

Figure 1. Number of Sessions Attended by Each Participant. Each box represents one attended session. The total number of sessions planned were 35 . 
Table 2. Comparison of Outcome Variables at Baseline and at $7^{\text {th }}$ Week

\begin{tabular}{lccc}
\hline & Baseline & $7^{\text {th }}$ week & p-value \\
\hline Diaphragm thickness (cm) & $0.35 \pm 0.038$ & $0.32 \pm 0.0317$ & 0.076 \\
Diaphragm mobility (quiet breathing) $(\mathrm{cm})$ & $2.17 \pm 0.61$ & $1.5 \pm 0.46$ & $0.025^{*}$ \\
Diaphragm mobility(deep breathing) $(\mathrm{cm})$ & $3.72 \pm 1.47$ & $2.58 \pm 0.48$ & $0.027^{*}$ \\
MIP (cm H20) & $53.5 \pm 17.7$ & $42.0 \pm 6.44$ & $0.047^{*}$ \\
MEP (cm H20) & $41.2 \pm 11.14$ & $34 \pm 2.98$ & 0.055 \\
FVC (L) & $1.57 \pm 0.25$ & $1.25 \pm 0.065$ & $0.002^{*}$ \\
FEV1(L) & $1.165 \pm 0.18$ & $0.99 \pm 0.13$ & $0.012^{*}$ \\
6 MWD (m) & $442 \pm 97.27$ & $212.6 \pm 89.16$ & $<0.001^{*}$ \\
\hline MIP, Max
\end{tabular}

MIP, Maximal Inspiratory Presssure; MEP, Maximal Expiratory Pressure; FVC, Forced Vital Capacity; FEV1, Forced Expiratory Volume in first second; 6 MWD, Six Minute Walk Distance; *, Significant at 5\% level of significance

discontinuation of research in view of the national lockdown guidelines and another participant died in the $5^{\text {th }}$ week of the treatment course due to collapse. 10 participants completed a total of 260 training sessions out of the 350 planned sessions denoting the adherence level as $74 \%$ as shown in Figure 1. However, no adverse events were reported during or following the intervention.

The changes in the outcome variables assessed at baseline and after 7 weeks of chemoradiation are represented in Table 2. At the end of 7 weeks, diaphragm thickness $(\mathrm{cm})$ showed no significant change from baseline $(p=0.076)$. Diaphragm mobility $(\mathrm{cm})$ which was evaluated using the ultrasound during quiet and deep breathing showed significant reduction from baseline to 7 th week $(p=0.025)$ and $(p=0.027)$ respectively. The MIP $\left(\mathrm{cmH}_{2} \mathrm{O}\right)$ showed changes from baseline to 7 weeks $(\mathrm{p}=0.047)$ while $\mathrm{MEP}\left(\mathrm{cmH}_{2} \mathrm{O}\right)$ showed no significant change $(p=0.055)$. The FVC and FEV1 both significantly reduced from baseline to $7^{\text {th }}$ week $(p=0.002)$ and $(\mathrm{p}=0.012)$ respectively. Also, statistically significant reduction was seen in the functional capacity among the participants $(\mathrm{p}<0.001)$.

\section{Discussion}

Inspiratory Muscle Training in known to alleviate pulmonary symptoms by improving respiratory mechanics and ventilatory reserves (Beaumont et al., 2018). However, there is lack of evidence studying a specific physiotherapy intervention addressing the cardiorespiratory complications following $\mathrm{HNC}$ and treatment related toxicities. Hence, this is the first pilot study to date assessing if inculcating an IMT program to the routine care of patients with $\mathrm{HNC}$ receiving CCRT would be feasible, adherent, and safe. We also evaluated if IMT program can influence diaphragm thickness, mobility, and various cardiorespiratory parameters in this population.

In spite the great advances in the field of exercise-based rehabilitation in cancer, there are very few studies examining the effects of exercise training in patients with HNC (D'Souza et al., 2020). Because of the high levels of treatment related toxicities causing barriers to exercise interventions, testing the feasibility of an intervention in such population is of prime importance before evaluating their efficacy in larger sample size. Our results indicate that an IMT intervention is not just feasible, but also well tolerated in patients with HNC receiving CCRT. Although a good recruitment rate $(76.9 \%)$ was reported in our study, participation was limited due to the ongoing viral pandemic. Despite this, our study showed an adherence rate of $74 \%$. However, as the treatment progressed, participants showed difficulty in performing the intervention in the last few days of CCRT. Treatment related complications such as pain, fever, fatigue, vomiting, generalised weakness, inadequate nutrition, oral mucositis were the barriers to the intervention during the later weeks. Participants who developed mild to moderate trismus in the later weeks of CCRT were able to perform the intervention with no adverse events reported implying the safety of the intervention. Some participants also reported that an IMT intervention along with the routine care improved their ability to expectorate but this was not evaluated objectively due to a lack of control group in this study.

A particular concern of patients with $\mathrm{HNC}$ receiving CCRT is the early manifestations of sarcopenia that reduce muscle mass and decrease the sarcomere contractility (Chauhan et al., 2020). A study done among elderly women who may have age-related sarcopenia showed significant improvement in diaphragm thickness, mobility, and respiratory muscle strength following an 8-week IMT program (Souza et al., 2014). Sarcopenia is a common aging process, but it has shown more pronounced adverse outcomes in patients with cancer. Due to the raised inflammatory response, metabolic alterations and significantly reduced physical activity levels in patients with cancer there is further decrement in lean body mass following CCRT (Ligibel et al., 2020). Hence, an IMT intervention could not possibly overcome these cancer related side effects, showing no significant change in diaphragm thickness $(p=0.076)$ but a significant reduction in diaphragm mobility $(\mathrm{p}=0.025$ and $\mathrm{p}=0.027)$ post 7 weeks of CCRT. Diaphragm function is positively correlated to respiratory muscle strength, pulmonary function, and functional capacity (Paulin et al., 2007; Rocha et al., 2017; Evrin et al., 2019). Diaphragmatic abnormality would decrease inspiratory muscle strength affecting the respiratory mechanics leading to reduced lung volumes and capacities (Roberts et al., 2013). This has been corroborated by the findings of our study where a significant reduction was seen in MIP $(p=0.047)$, 
FVC $(p=0.002)$ and FEV1 $(p=0.012)$ measures. Also, loss of diaphragm function causes a potential mismatch between the activation of phrenic nerve and generation of diaphragmatic force. This could result in breathlessness, difficulty in sustaining higher ventilation required for physical activity, and ultimately leading to exercise intolerance, as shown by a decline of 6-MWD $(p<0.001)$ in our study (Fogarty et al., 2018). However, MEP showed no significant change $(p=0.055)$. Although there were no morphologic changes, recruitment of motor units (neural adaptation) could be the speculated mechanism that did not cause a decline in expiratory muscle strength following an IMT intervention. This was also indicated by a subjective improvement in the cough effort It is also interesting to note that participants did feel symptomatically better which was recorded on Borg Scale used for progressing the load intensity of IMT. However, the small number of participants may limit our ability to see changes in the physiologic parameters.

Although no profound effect of IMT was seen on the various cardiorespiratory parameters in this study, we demonstrated our primary outcome that a threshold IMT device is feasible, safe, and well tolerated by the patients with advanced stage HNC receiving CCRT. Secondary outcome and potential benefits of using IMT in patients receiving concomitant chemoradiation can only be evaluated in larger sample and using randomised study designs. Also, it is important to evaluate these outcomes as IMT can be used as preventive support in the long-term manifestations of cancer related side effects.

In conclusion, this is one of the first studies to examine the role of IMT in patients with head and neck caner undergoing IMT. IMT in patients undergoing CCRT for head and neck cancer, was feasible, safe, and well tolerated by the patients as indicated by a good adherence level. Further studies with randomised controlled trials with a larger sample size are required to reveal the potential effects of IMT during CCRT in head and neck cancer patients.

\section{Author Contribution Statement}

The idea for the study was formulated by Dr Stephen, Ms Prina and Dr Balachandran, methodological direction was given by Dr Sampath, ultrasonography was done by Dr Santosh Rai and collected data was analysed by Dr Ravishankar. All the authors equally contributed for writing and editing the manuscript. Any data regarding the study will be made available by the authors on request.

\section{Acknowledgments}

The study was a part of an approved student thesis for master's program. It was approved by Institutional Ethics Committee (IEC KMC MLR 11-19/572). No funding was provided for the study and there was no conflict of interest.

\section{References}

Beaumont M, Forget P, Couturaud F, et al (2018). Effects of inspiratory muscle training in COPD patients: A systematic review and meta-analysis. Clin Respir J, 12, 2178-88.

Chauhan NS, Samuel SR, Meenar N, et al (2020). Sarcopenia in male patients with head and neck cancer receiving chemoradiotherapy: a longitudinal pilot study. Peer $J, \mathbf{8}$, e8617.

D’Souza M, Samuel SR, Rai SP, et al (2020). Respiratory muscle function and exercise capacity in a stage 4 head and neck cancer patient receiving chemoradiotherapy. Indian J Palliat Care, 26, 385.

D'Souza M, Samuel SR, Saxena PP (2020). Effects of exercise training during concomitant chemoradiation therapy in headand-neck cancer patients: A Systematic Review. Indian $J$ Palliat Care, 26, 531-2.

Davis MP, Panikkar R (2019). Sarcopenia associated with chemotherapy and targeted agents for cancer therapy. Ann Palliat Med, 8, 86-101.

De Almeida LB, Trevizan PF, Laterza MC, et al (2020). Safety and feasibility of inspiratory muscle training for hospitalized patients undergoing hematopoietic stem cell transplantation: a randomized controlled study. Support Care Cancer, 28, 3627-35.

Dina Brooks, Sherra Solway, William J Gibbons (2003). ATS statement: guidelines for the six-minute walk test. $\mathrm{Am} \mathrm{J}$ Respir Crit Care Med, 167, 1287.

Evrin T, Korkut S, Ozturk Sonmez L, et al (2019). Evaluating stable chronic obstructive pulmonary disease by ultrasound. Emerg Med Int, 2019, 5361620.

Fields DP, Roberts BM, Simon AK, et al (2019). Cancer cachexia impairs neural respiratory drive in hypoxia but not hypercapnia. J Cachexia Sarcopenia Muscle, 10, 63-72.

Fogarty MJ, Mantilla CB, Sieck GC (2018). Breathing: Motor Control of Diaphragm Muscle. Physiology (Bethesda), 33, 113-26.

Frownfelter D (1987). Principles and practice of cardiopulmonary physical therapy.In ' Respiratory Muscle Weakness and Training', Elsevier health sciences, Philadelphia, pp 448-9.

Gottlieb M, Marsaa K, Godtfredsen NS, et al (2015). Prevalence and management of pulmonary comorbidity in patients with lung and head and neck cancer. Acta Oncol, 54, 767-71.

Graham BL, Steenbruggen I, Miller MR, et al (2019). Standardization of spirometry 2019 update. An official American thoracic society and European respiratory society technical statement. Am J Respir Crit Care Med, 200, e70-e88.

Kelley RC, Ferreira LF (2017). Diaphragm abnormalities in heart failure and aging: mechanisms and integration of cardiovascular and respiratory pathophysiology. Heart Fail Rev, 22, 191-207.

Lai Y, Huang J, Yang M, et al (2017). Seven-day intensive preoperative rehabilitation for elderly patients with lung cancer: a randomized controlled trial. J Surg Res, 209, 30-6.

Ligibel JA, Schmitz KH, Berger NA (2020). Sarcopenia in aging, obesity, and cancer. Transl Cancer Res, 9, 5760.

Merrell AJ, Kardon G (2013). Development of the diaphragm -- a skeletal muscle essential for mammalian respiration. Febs $J, 280,4026-35$.

Nakano J, Ishii S, Fukushima T, et al (2017). Factors affecting muscle strength in cancer patients receiving chemotherapy. J Nov Physiother Rehabil, 1, 56-66.

Paulin E, Yamaguti WP, Chammas MC, et al (2007). Influence of diaphragmatic mobility on exercise tolerance and dyspnea in patients with COPD. Respir Med, 101, 2113-8.

Petrar S, Bartlett C, Hart RD, et al (2012). Pulmonary complications after major head and neck surgery: A retrospective cohort study. Laryngoscope, 122, 1057-61.

Roberts BM, Ahn B, Smuder AJ, et al (2013). Diaphragm and ventilatory dysfunction during cancer cachexia. Faseb $J$, 
27, 2600-10.

Rocha FR, Brüggemann AK, Francisco DS, et al (2017). Diaphragmatic mobility: relationship with lung function, respiratory muscle strength, dyspnea, and physical activity in daily life in patients with COPD. J Bras Pneumol, 43, 32-7.

Santana A, Caruso P, Santana PV, et al (2018). Inspiratory muscle weakness, diaphragm immobility and diaphragm atrophy after neck dissection. Eur Arch Otorhinolaryngol, 275, 1227-34.

Silverman DA, Lin C, Tamaki A, et al (2020). Respiratory and pulmonary complications in head and neck cancer patients: Evidence-based review for the COVID-19 era. Head Neck, 42, 1218-26.

Souza H, Rocha T, Pessoa M, et al (2014). Effects of inspiratory muscle training in elderly women on respiratory muscle strength, diaphragm thickness and mobility. J Gerontol A Biol Sci Med Sci, 69, 1545-53.

Steier J, Kaul S, Seymour J, et al (2007). The value of multiple tests of respiratory muscle strength. Thorax, 62, 975-80.

Testa A, Soldati G, Giannuzzi R, et al (2011). Ultrasound M-mode assessment of diaphragmatic kinetics by anterior transverse scanning in healthy subjects. Ultrasound Med Biol, 37, 44-52.

Ueki J, De Bruin P, Pride N (1995). In vivo assessment of diaphragm contraction by ultrasound in normal subjects. Thorax, 50, 1157-61.

Van Adrichem EJ, Meulenbroek RL, Plukker JT, et al (2014). Comparison of two preoperative inspiratory muscle training programs to prevent pulmonary complications in patients undergoing esophagectomy: a randomized controlled pilot study. Ann Surg Oncol, 21, 2353-60.

Volpe RC, Fitipaldi R, Silva RA, et al (2011). [Association of chronic pulmonary obstructive disease (COPD) and complications in head and neck surgery]. Braz $J$ Otorhinolaryngol, 77, 125-8.

Wenzel JA, Griffith KA, Shang J, et al (2013). Impact of a home-based walking intervention on outcomes of sleep quality, emotional distress, and fatigue in patients undergoing treatment for solid tumors. Oncologist, 18, 476-84.

\section{๑๐요}

This work is licensed under a Creative Commons AttributionNon Commercial 4.0 International License. 